Eur J Clin Chem Clin Biochem

1995; 33:95-98

(c) 1995 Walter de Gruyter \& Co.

Berlin · New York

\title{
Distinction Between Homozygous and Heterozygous Subjects with Hereditary Haemochromatosis Using Iron Status Markers and Receiver Operating Characteristic (ROC) Analysis
}

\author{
By Nils Milman' and Michael J. Albeck \\ 1 Department of Medicine Y, Gentofte Hospital, University of Copenhagen, Copenhagen, Denmark \\ 2 University Clinic of Neurosurgery, Rigshospitalet, Copenhagen, Denmark
}

(Received August 25/December 8, 1994)

Summary: The purpose of the present study was:

1) to evaluate which of the four iron status markers (serum iron, serum transferrin, serum transferrin saturation, serum ferritin) displayed the highest discriminatory potential in the distinction between homozygous patients with hereditary haemochromatosis and heterozygous relatives,

2) to suggest optimum cut-off values for these iron status markers, and

3) to demonstrate how these cut-off values change if the expected utility from a correct diagnosis is incorporated into the analysis.

The patients and relatives were found by a nation-wide epidemiological survey. The study population consisted of 162 patients with clinically overt hereditary haemochromatosis and 84 asymptomatic heterozygous relatives. The statistical evaluation was performed using receiver operating characteristic (ROC) curve analysis. The diagnostic power of the iron markers is expressed as the area under the ROC curve. The optimum cut-off value is at the point where the slope of the ROC curve is equal to one. Changes in the optimum cut-off value at varying expected utility from a correct classification was estimated by changing the scaling of the ROC diagram. Serum iron and serum transferrin had the smallest area under the ROC curve, and were both unsuitable as discriminators. Near complete discrimination was obtained with serum transferrin saturation and serum ferritin concentrations, displaying the largest area under the ROC curve ( 0.991 and 0.998$)$. The optimum threshold value for transferrin saturation was $61 \%$, and for serum ferritin concentration $800 \mu \mathrm{g} / \mathrm{l}$. The transferrin saturation level reflects the presence of the haemochromatosis allele, whereas the serum ferritin concentration indicates the degree of iron overload. Changes in the optimum cut-off value from varying expected utility implicated the importance of incorporating this discriminator.

\section{Introduction}

Hereditary haemochromatosis is a disorder of iron metabolism, characterized by intestinal hyperabsorption of iron, parenchymal iron overload and subsequent organ damage $(1,2)$. Much effort has been devoted to the search for a reliable non-invasive test of preclinical and early haemochromatosis, but so far the best discriminators are still biochemical iron status markers, serum transferrin saturation and serum ferritin concentration
$(1-6)$. There is virtually no overlapping of transferrin saturation in subjects being homozygous $(h / h)$ for the haemochromatosis allele and normal $(-/-)$ subjects, whereas discriminatory problems exist between homozygous and heterozygous $(\mathrm{h} / \mathrm{-})$ subjects $(3-6)$. The present study uses receiver operating characteristic (ROC) curve analysis in the discrimination between subjects being homozygous and heterozygous for the haemochromatosis allele. 


\section{Subjects and Methods}

The material consisted of samples from 162 Danish patients (126 men, 36 women, median age 55 years, range 29-81) with hereditary haemochromatosis, found by a nation-wide epidemiological survey $(2,6)$. All patients had clinically overt disease and were considered to be homozygous for the haemochromatosis allele. The diagnostic criteria and the selection of the patients have been described previously $(2,6)$. Pedigree studies were performed in 32 of the patients (probands) with haemochromatosis, HLA-typing being employed in 21 families (7). Altogether, 84 heterozygous subjects ( 39 men, 45 women) were identified and included in the study.

Blood samples were obtained in the fasting state in the morning. Serum iron was analyzed by spectrophotometry on a centrifugal analyser (Cobas Bio ${ }^{\circledR}, \mathrm{F}$. Hoffmann-La Roche AG, CH-4002 Basel) using guanidinium ferrozine as chromogen, following release from transferrin with $\mathrm{HCl}$ and subsequent reduction with ascorbic acid (Unimate 7 Iron ${ }^{\circledR}$, Roche Diagnostic Systems, F. HoffmannLa Roche AG, CH-4002 Basel). Samples were analyzed in duplicate. The overall analytical coefficient of variation was $3.1 \%$.

Serum transferrin (IFCC relative molecular mass $M_{\mathrm{r}} 74000$ ) was measured by immunoturbidimetry on a centrifugal analyser (Cobas $\mathrm{Bio}^{\circledR}$, F. Hoffmann-La Roche AG, CH-4002 Basel) using the Unimate 3 Transferrin ${ }^{\circledR}$ (Roche Diagnostic Systems, F. Hoffmann-La Roche AG, CH-4002 Basel). Calibration was performed with the human protein standard CRM 470. Samples were analysed in duplicate. The overall analytical coefficient of variation was $3.3 \%$. The transferrin saturation in percent was calculated as: serum iron, $\mu \mathrm{mol} / \mathrm{l} \times 100 /$ serum transferrin, $\mu \mathrm{mol} / 1 \times 2$.

Serum ferritin was measured with a two-site immunoradiometric assay, employing specially prepared paper discs as the solid phase (Phadebas Ferritin PRIST ${ }^{\circledR}$, Pharmacia Diagnostics AB, S-75182 Uppsala) and described in detail elsewhere (8). Samples were run in duplicate. The overall analytical coefficient of variation was $8.8 \%$. Calibration of the kit using the WHO Human Liver Ferritin International Standard 80/602, showed that a Phadebas value of 11 $\mu \mathrm{g} / \mathrm{l}$ corresponded to a WHO value of $15 \mu \mathrm{g} / \mathrm{l}(9)$.

\section{Statistical methods}

In order to obtain an estimate of the diagnostic power of the four variables, ROC curves were constructed. A ROC curve is a plot of the true positive fraction, TPF, (n (true positive)/n (all diseased subjects)), as a function of the false positive fraction, FPF, (n (false positive)/n (all non-diseased subjects)), when the cut-off value runs from $-\infty$ to $+\infty(10)$. The ROC curves were fitted by the equation (11)

$$
\mathrm{TPF}=\mathrm{R} \times \mathrm{FPF}^{1 / \mathrm{E}}+(1-\mathrm{R}) \times\left(1-(1-\mathrm{FPF})^{\mathrm{E}}\right)
$$

where $\mathrm{E}$, which is $>1$ for any test of practical value, determines the height of the vault of the curve, and $R$ the skewness of the curve $(0 \leqslant R \leqslant 1)$. The $E$ and $R$ parameters were estimated by non-linear regression. A least square lost function was used. The minimization algorithm was initiated by a Rosenbrock algorithm (to avoid local minima), followed by a quasi-Newton minimization algorithm (12). The area under the ROC curve was subsequently calculated as

$$
\text { Area }=E /(E+1)
$$

and was taken as an estimate of accuracy.

The optimum cut-off value is situated at the point where the slope of the ROC curve is equal to one. To include the expected utility from a correct classification, the axes were scaled by the frequencies of the two conditions (homozygosity and heterozygosity) and the expected utility. The ordinate axis was multiplied by the frequency of homozygous subjects and by the benefit a homozygous patient would miss if he was misclassified, i. e., false negative. The abscissa axis was multiplied by the frequency of heterozygous subjects and by the cost of examining a non-diseased per- son (13). The cut-off values were calculated for various benefit/ cost ratios.

\section{Results}

Iron status markers in homozygous patients and heterozygous subjects are shown in table 1. Homozygotes had significantly higher serum iron concentration, lower serum transferrin concentration, higher transferrin saturation, and higher serum ferritin concentration than heterozygotes.

The ROC curves for the four iron status markers are depicted in figure 1, and the area under the ROC curve, and optimum discriminatory values for each variable are displayed in table 2 .

It is evident from the curve that serum iron concentration and serum transferrin concentration had the lowest discriminatory potential between homozygous and het-

Tab. 1 Iron status markers (median and range) in homozygous patients with clinical hereditary haemochromatosis, and in healthy heterozygous subjects.

\begin{tabular}{lllll}
\hline & $\begin{array}{l}\text { Serum } \\
\text { iron } \\
(\mu \mathrm{mol} / \mathrm{l})\end{array}$ & $\begin{array}{l}\text { Serum } \\
\text { transferrin } \\
(\mu \mathrm{mol} / \mathrm{l})\end{array}$ & $\begin{array}{l}\text { Serum } \\
\text { transferrin } \\
\text { saturation } \\
(\%)\end{array}$ & $\begin{array}{l}\text { Serum } \\
\text { ferritin }\end{array}$ \\
$(\mu \mathrm{g} / \mathrm{l})$ \\
\hline $\begin{array}{l}\text { Homo- } \\
\text { zygotes } \\
\mathrm{n}=162\end{array}$ & $39^{*}$ & $22^{*}$ & $87^{*}$ & $3400^{*}$ \\
$\begin{array}{l}\text { Hetero- } \\
\text { zygotes } \\
\mathrm{n}=84\end{array}$ & $19-61$ & $9-36$ & $52-100$ & $800-12700$ \\
\hline
\end{tabular}

*) Mann-Whitney test: $\mathrm{p}<0.0001$

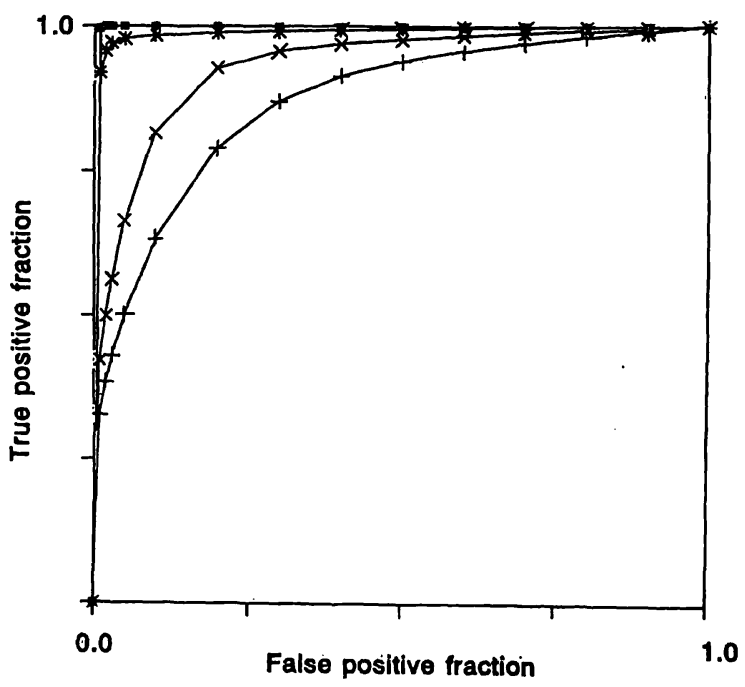

Fig. 1 Exponentially fitted receiver operating characteristic (ROC) curves for iron status markers in hereditary haemochromatosis.
$\times$ S-iron
+ S-transferrin
* Transferrin saturation
ธ. S-ferritin 
Tab. 2 Optimum cut-off values for iron status markers in hereditary haemochromatosis at various levels of expected utility. The diagnostic power of the four markers are expressed as the area under the ROC curve. B denotes the benefit a homozygous patient would miss if he was misclassified, i. e., false negative. $C$ denotes the cost of examining a non-diseased person. According to different levels of expected utility, corresponding cut-off values for various $B / C$ ratios are shown (see text).

\begin{tabular}{llllll}
\hline Iron status marker & $\begin{array}{l}\text { Area } \\
\text { under the } \\
\text { ROC } \\
\text { curve }\end{array}$ & $\begin{array}{l}\text { Cut-off value } \\
\text { B/C }=\end{array}$ & $1 / 1$ & $1 / 10$ & $1 / 100$ \\
\hline & 0.935 & 30 & 18 & 16 \\
\hline $\begin{array}{l}\text { Serum iron } \\
(\mu \mathrm{mol} / 1)\end{array}$ & 0.874 & 34 & 36 & 40 \\
$\begin{array}{l}\text { Serum transferrin } \\
(\mu \mathrm{mol} / \mathrm{l})\end{array}$ & 0.991 & 61 & 44 & 44 \\
$\begin{array}{l}\text { Serum transferrin } \\
\text { saturation }(\%)\end{array}$ & 0.998 & 800 & & \\
$\begin{array}{l}\text { Serum ferritin } \\
(\mu \mathrm{g} / \mathrm{l})\end{array}$ & & & & & \\
\hline
\end{tabular}

erozygous subjects. The optimum cut-off value was 30 $\mu \mathrm{mol} / \mathrm{l}$ for serum iron and $34 \mu \mathrm{mol} / \mathrm{l}$ for serum transferrin (tab. 2).

Transferrin saturation and serum ferritin concentration had far better discriminatory abilities. Almost complete discrimination was obtained with these two variables, displaying the largest area under the ROC curve. The optimum cut-off value was $61 \%$ for transferrin saturation and $800 \mu \mathrm{g} / 1$ for serum ferritin concentration (tab. 2).

\section{Discussion}

Until specific DNA-probes which can detect the haemochromatosis allele have been developed, the available discriminators consist of the biochemical tests serum transferrin saturation and serum ferritin concentration. Due to the biological variation, it is often difficult to define the threshold value, by which the clinician will be able to discriminate between normal and abnormal. The ROC analysis has been developed to cope specifically with this problem.

It appeared that both serum iron concentration and serum transferrin concentration were unsuitable as discriminators in haemochromatosis, both displaying a high degree of misclassification. Similar results have been reported in other studies $(3,4,6)$.

Serum ferritin concentration and transferrin saturation displayed the highest discriminatory ability, with an optimum calculated value for transferrin saturation of $61 \%$. The diagnostic potential of transferrin saturation has been evaluated by other statistical methods $(3-6) . D a-$ done et al. (3) examined laboratory data in pedigree studies of haemochromatosis patients, using discriminant analysis, and found that transferrin saturation was superior to serum ferritin concentration in predicting the genotype. A threshold transferrin saturation value of $62 \%$ correctly predicted homozygosity in $92 \%$ of the cases (3).

Borwein et al. (5) evaluated the diagnostic potential of transferrin saturation alone and in combination with serum ferritin concentration in pedigree studies using a likelihood analysis model. A transferrin saturation of $55 \%$ provided the best statistical combination of truepositive and true-negative results. A cut-off point of $55 \%$ for transferrin saturation and a cut-off point at the 90th percentile for serum ferritin concentration were sufficient for detection of haemochromatosis, when the results of one or both tests were positive (5).

Bassett et al. (4) assessed the value of transferrin saturation and serum ferritin concentration using sensitivity, specificity, predictive value of a positive test $\left(\mathrm{PV}_{\mathrm{pos}}\right)$ and predictive value of a negative test $\left(P V_{\text {neg }}\right)$. Transferrin saturation and serum ferritin concentration demonstrated high levels of sensitivity and specificity. The authors suggested a cut-off point of $55 \%$ for transferrin saturation. The diagnostic efficiency was not improved if transferrin saturation and serum ferritin concentration were combined. However, serum ferritin concentration appeared to be an accurate indicator of body iron overload (4).

In a previous report (6), the present series was analyzed using predictive values, $\mathrm{PV}_{\text {pos }}, \mathrm{PV}_{\text {neg }}$, and efficiency according to Vecchio (14). The maximum efficiency for the four iron status markers was obtained with the following discriminatory values: serum iron, $29 \mu \mathrm{mol} / \mathrm{l}$; serum transferrin, $30 \mu \mathrm{mol} / \mathrm{l}$; transferrin saturation, $60 \%$; serum ferritin, $800 \mu \mathrm{g} / \mathrm{l}(6)$. Clearly, the cut-off values calculated by ROC analysis were similar or close to those calculated by the Vecchio principles.

Three different statistical methods, discriminant analysis (3), predictive analysis according to Vecchio (6) and ROC analysis have indicated a transferrin saturation threshold value of $60-62 \%$ as being most efficient in the discrimination between subjects being homozygous and heterozygous for the haemochromatosis allele.

ROC analysis gives the opportunity to adjust for the lost benefit of false negative and cost of false positive misclassifications. Untreated haemochromatosis may lead to premature death, most often due to cirrhosis of the liver and/or hepatoma $(1,2)$. If the genetic abnormality is recognized in the preclinical phase, overt disease can be prevented by depletion of the iron overload through therapeutic phlebotomy. 
The disease prevalence of hereditary haemochromatosis among Danish male blood donors is estimated to 0.37$0.46 \%$ (15). The ultimative cost of a false negative misclassification may be permanent disability and premature death, whereas the cost of a false positive misclassification includes the expense of an additional serum ferritin measurement and a liver biopsy.

The transferrin saturation level reflects the presence of the haemochromatosis allele, while the serum ferritin concentration reflects the level of iron overload. Serum ferritin concentration per se cannot therefore be used in the general screening procedure, as subjects with preclinical haemochromatosis may have normal or only slightly elevated concentrations. When a transferrin saturation above the critical level has been identified, serum ferritin concentration should be measured in order to assess the magnitude of the iron overload, i.e., the severity of the disease. The lowest ferritin concentration found in homozygous subjects with clinically overt disease in this series was $800 \mu \mathrm{g} / \mathrm{l}$, while homozygous subjects with preclinical disease display values ranging from 50 to $600 \mu \mathrm{g} / \mathrm{l}$ (2). According to the present results, a critical serum ferritin value of $800 \mu \mathrm{g} / \mathrm{l}$ seems appropriate to discriminate between patients with preclinical and clinical disease.

\section{Acknowledgement}

The study was supported by grants from the Research Foundation of the Danish Medical Association and the Health Insurance Foundation (grant no. H 11-4-87 and H 11-15-89). The authors are indebted to Jørgen Hilden M.D., Statistical Research Unit, University of Copenhagen, Denmark, for critical revison of the manuscript.

\section{References}

1. Edwards CQ, Dadone M, Skolnick MH, Kushner JP. Hereditary haemochromatosis. Clin Haematol 1982; 11:411-36.

2. Milman N. Hereditary haemochromatosis in Denmark 19501985. Clinical, biochemical and histological features in 179 patients and 13 preclinical cases. Dan Med Bull 1991; 38:385-93.

3. Dadone MM, Kushner JP, Edwards CO, Bishop DT, Skolnick $\mathrm{MH}$. Hereditary hemochromatosis. Analysis of laboratory expression of the disease by genotype in 18 pedigrees. Am J Clin Pathol 1982; 78:196-207.

4. Bassett ML, Halliday JW, Ferris RA, Powell LW. Diagnosis of hemochromatosis in young subjects: Predictive accuracy of biochemical screening tests. Gastroenterology 1984; 87:62833.

5. Borwein S, Ghent CN, Valberg LS. Diagnostic efficacy of screening tests for hereditary hemochromatosis. Can Med Assoc J 1984; 131:895-901.

6. Milman N. Iron status markers in hereditary haemochromatosis: distinction between individuals being homozygous and heterozygous for the haemochromatosis allele. Eur $\mathrm{J}$ Haematol 1991; 47:292-8.

7. Milman N, Graudal N, Nielsen LS, Fenger K. A HLA study in 74 Danish haemochromatosis patients and in 21 of their families. Clin Genet 1992; 41:6-11.

8. Milman N, Pedersen NS, Visfeldt J. Serum ferritin in healthy Danes: relation to marrow haemosiderin iron stores. Dan Med

Bull 1983; 30:115-20.
9ilman N, Graudal N, Juul-Jørgensen B, Bentzon MW. Calibration of the Amersham Ferritin RIA Kit using the WHO Human Liver Ferritin International Standard 80/602. Eur J Clin Chem Clin Biochem 1994; 32:41-2.

10. Metz CE. Basic principles of ROC analysis. Semin Nucl Med 1978; 8:283-98.

11. England WL. An exponential model used for optimum threshold selection on ROC curves. Med Decis Making 1988; 8:120-31.

12. CSS: Statistica. Statsoft Inc., Tulsa 1991: vol 2:423-52.

13. Hilden J. The area under the ROC curve and its competitors. Med Decis Making 1991; 11:95-101.

14. Vecchio TJ. Predictive value of a single diagnostic test in unselected populations. N Engl J Med 1966; 274:1171-3.

15. Wiggers P, Dalhøj J, Kiær H, Ring-Larsen H, Petersen PH, Blaabjerg O, Hørder M. Screening for haemochromatosis: prevalence among Danish blood donors. J Intern Med 1991; 230:265-70.
Dr. Nils Milman
Department of Medicine
Øresund Hospital
DK-2970 Hørsholm
Denmark 